\title{
ON STRONG MEASURABILITY OF BANACH VALUED FUNCTIONS ${ }^{1}$
}

\author{
MICHEL MÉTIVIER
}

The purpose of this note is to point out that, as an immediate consequence of our Theorem 11 in [3], we get an interesting measurability property for some Banach valued functions.

As a corollary, we get an improvement of Theorem 4 of [3], to the extent that the separability condition in [3] can be dropped.

1. Statement of the Theorem. Let $(\Omega, \mathfrak{F}, \mu)$ be a measure space, $\mu$ being a finite positive measure on the $\sigma$-field $\mathfrak{F}$ of subsets of $\Omega$. Let $B$ be a Banach space, and $\subseteq$ a set of convex equilibrated weakly compact subsets of $B$. A mapping $f$ from $\Omega$ into $B$ is said to be strongly $\mu$ measurable if it takes its values in a separable subset of $\boldsymbol{B}$ and, for every ball $B$ in $B, f^{-1}(B)$ belongs to the $\mu$-completion of $\mathfrak{F}$. We denote by $\boldsymbol{B}^{\prime}$ the Banach dual space of $\boldsymbol{B}$.

Then we have the following

THeOREM. Let $f$ be a mapping of $(\Omega, \mathfrak{F}, \mu)$ into the Banach space $B$, with the following properties:

(i) For every strictly positive number $\epsilon$, there exists $\Omega_{\epsilon} \in \mathfrak{F}$ and $Q_{\epsilon} \in \mathfrak{S}$ such that $\mu\left(\Omega \backslash \Omega_{\epsilon}\right) \leqq \epsilon, f\left(\Omega_{\epsilon}\right) \subset Q_{\epsilon}$.

(ii) For every continuous linear form $x^{\prime}$ on $B$, the mapping $\omega \hookrightarrow x^{\prime} \circ f(\omega)$ (denoted $\left\langle f, x^{\prime}\right\rangle$ ) from $(\Omega, \mathfrak{F}, \mu)$ into $R$ (set of real numbers) is $\mu$-measurable.

Then, there exists a strongly $\mu$-measurable mapping $\bar{f}$ from $(\Omega, \mathfrak{F}, \mu)$ into $B$, such that for every $x^{\prime} \in B^{\prime},\left\langle f, x^{\prime}\right\rangle=\left\langle\bar{f}, x^{\prime}\right\rangle \mu$ a.e.

2. Proof of the Theorem. For every $F \in \mathfrak{F}$, with $F \subset \Omega_{\epsilon}$, it is well known (cf. [1] for example) that the weak integral $\int_{F} f d \mu$ exists and

$$
m(F)=\int_{F} f d \mu \in \mu(F) Q_{\epsilon} .
$$

Let us then apply Theorem 11 of [3] to the restriction of $\mu$ and $m$ to $\Omega_{\epsilon}$. We see then that there exists a strongly measurable $\bar{f}_{\mathrm{e}}$ such that $m(F)=\int_{F} \bar{f}_{\epsilon} d \mu$ for every $F \subset \Omega_{\epsilon}$. Hence, for every $x^{\prime} \subset B^{\prime}$ we have $\left\langle\bar{f}_{\epsilon}, x^{\prime}\right\rangle=\left\langle f, x^{\prime}\right\rangle$ a.e. on $\Omega_{\epsilon}$. The theorem follows by considering a sequence $\Omega_{n}$ of disjoint subsets of $\Omega$, such that $\mu\left(\cup_{n} \Omega_{n}\right)=\mu(\Omega)$ and defining $\bar{f}$ by its restriction $\bar{f}_{n}$ to each $\Omega_{n}$.

Received by the editors June 10, 1968.

1 This research was supported by a grant from the National Science Foundation, under contract No. GP-4867. 
3. Application to Banach valued martingales. Let us go back to the terminology of [3], considering the strong martingale $\left(\mathfrak{F}_{\alpha}, f_{\alpha}\right)_{\alpha \in I}$ as it is described by the assumptions of Theorem 4, but dropping the separability assumption on the Banach space $V$. The function $f_{\infty}$ which occurs at the beginning of the proof in [3] verifies the assumptions of the above theorem, and can be then replaced by a $\bar{f}_{\infty}$ which is strongly measurable and possesses the same properties with respect to the martingale $\left(\mathfrak{F}_{\alpha}, f_{\alpha}\right)_{\alpha \in I}$.

In the special case when $V$ is reflexive, we can thus state

CoROllaRY. Let $\left(\mathfrak{F}_{\alpha}, f_{\alpha}\right)_{\alpha \in I}$ be a strong martingale with values in a reflexive Banach space $V, I$ being any directed ordered set. In order for $\left(f_{\alpha}\right)_{\alpha \in I}$ to converge in $L^{1}(\Omega, \mathfrak{F}, \mu, V)$ to a strongly integrable function $f_{\infty}$, it is necessary and sufficient that the family $\left(f_{\alpha}\right)$ be terminally uniformly integrable.

\section{BIBLIOGRAPHY}

1. N. Bourbaki, Intégration, Chapter VI, Hermann, Paris, 1959.

2. N. Dunford and J. T. Schwartz, Linear operators, Part I, Interscience, New York, 1958.

3. M. Métivier, Martingales a valeurs vectorielles. Application à la dérivation des mesures vectorielles, Ann. Inst. Fourier Grenoble 2 (1967), 175-208.

Cornell University and

UNIVERSITY OF RENNES 